\title{
Robust dual reconstruction systems and fusion frames
}

\author{
P. G. Massey, M. A. Ruiz and D. Stojanoff \\ Depto. de Matemática, FCE-UNLP, La Plata, Argentina and IAM-CONICET
}

\begin{abstract}
We study the duality of reconstruction systems, which are $g$-frames in a finite dimensional setting. These systems allow redundant linear encoding-decoding schemes implemented by the so-called dual reconstruction systems. We are particularly interested in the projective reconstruction systems that are the analogue of fusion frames in this context. Thus, we focus on dual systems of a fixed projective system that are optimal with respect to erasures of the reconstruction system coefficients involved in the decoding process. We consider two different measures of the reconstruction error in a blind reconstruction algorithm. We also study the projective reconstruction system that best approximate an arbitrary reconstruction system, based on some well known results in matrix theory. Finally, we present a family of examples in which the problem of existence of a dual projective system of a reconstruction system of this type is considered.
\end{abstract}

keywords: Reconstruction systems, dual reconstruction systems, erasures, fusion frames. subclass: $42 \mathrm{C} 15$ and $15 \mathrm{~A} 60$.

\section{Contents}

1 Introduction

2 Basic framework of reconstruction systems

3 Optimal erasures and errors 6

3.1 Minimizing the 2 -error . . . . . . . . . . . . . . . . 7

3.2 Minimizing the worst-case reconstruction error . . . . . . . . . . . 9

4 Stability of RS's under erasures of coefficient packets 11

5 Approximation by projective RS's $\quad 12$

6 Examples $\quad \mathbf{1 4}$

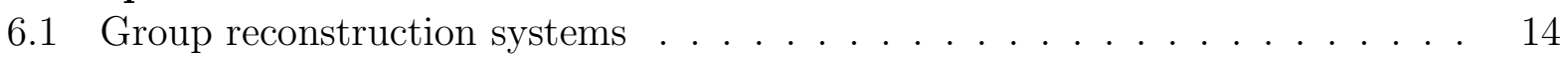

6.2 Dual projective systems . . . . . . . . . . . . . . . . . . . . . . . . . . . . . . . .

6.3 Riesz reconstruction systems . . . . . . . . . . . . . . . . 17 


\section{Introduction}

Many researchers have recently studied the problem of designing finite frames for the reconstruction of a signal $x$ in the absence of a number of (missing or delayed) coefficients (see [4, 5, 6, 17, 11, 12, 15, 19]). In this context, the goal is to construct finite frames which minimize the (blind) reconstruction error of a signal when a number $k$ of frame coefficients are "erased".

In [14, the authors study the following problem: given a fixed frame $\mathcal{F}=\left\{f_{i}\right\}_{i=1}^{m}$ for $\mathbb{C}^{d}$, find the alternate dual frames that minimize a measure of the (blind) reconstruction error when $k$ frame coefficients are erased. Thus, unlike the previous setting, the interest is centered in a subset of the set of alternate dual frames of a fixed frame. As they notice, the canonical dual is not always the best choice despite the fact that it is associated to the Moore-Penrose pseudoinverse. Nevertheless, one of the main results in [14] exhibits conditions on the fixed frame $\mathcal{F}$ which assure that the only optimal dual of $\mathcal{F}$ - in the sense previously described - is its canonical dual.

Our research is in the vein of [14] but our interest is to set it in the context of fusion frames. However, since the definition of fusion frames depends strongly on the subspaces of the frame, the concept of dual fusion frame is not easy to handle. Therefore, we identify fusion frames with a subset of a broader class, namely the Reconstruction Systems (RS) (finite dimensional $g$-frames, using the terminology of W. Sun [20]). Hence we fix a projective RS $\mathcal{V}$ (i.e. the analogue of fusion frames in the context of RS's), and we search for conditions that allow us to describe the dual RS's of $\mathcal{V}$ that minimize some measure of the (blind) reconstruction error.

As in [14, our results show the existence of a unique dual RS (which is not necessarily projective) that is optimal for the erasure of 1 packet of coefficients. As in previous works in the subject, the error is measured in terms of the Frobenius norms of the so-called "packet lost operators" (the equivalent notion of the error operators in [4, 11, 14]). We present two different ways to perform this measure: the worst case error (WCE), i.e. the largest packet lost operator norm among all possible erasures, and the 2-error which is the euclidean norm of the vector of all packet lost operator norms. Each of these errors bound the norm of the error when a vector $x$ is reconstructed with a blind reconstruction strategy, assuming that a frame coefficient is missing.

For the WCE, following the approach of Han and Lopez, we impose some conditions on the norms involving the canonical dual elements to assure that the canonical dual is the only optimal alternate dual for one erasure. For the 2-error we show that, for projective RS's, there is always a unique optimal dual. In both cases, our results include a detailed description of the optimal dual RS.

The fact that the optimal alternate duals of the fixed projective $\mathrm{RS} \mathcal{V}$ considered above need not be projective motivates the study of best projective approximations of an arbitrary RS. We consider this problem in terms of well known results in matrix theory.

On the other hand, assuming that the erased packets of coefficients correspond to a known subset of indexes of the RS $\mathcal{V}$, we find conditions which assure that the RS corresponding to the remaining set of indexes is also a RS. Notice that this last fact allows to have perfect reconstruction with suitable dual RS's. We have included a section of examples in which we also consider the problem of the existence of projective duals of a given projective RS.

In order to put our results in perspective and to keep the text self contained we have reviewed some known results concerning the structure of optimal duals in the context of fusion frames and $g$-frames, including short proofs in some cases. It is worth pointing out 
that some results in $g$-frames theory can be obtained from their analogues in frame theory, using the fact that $g$-frames can be considered as groupings of vectors of a frame. We have used this kind of argument whenever it was possible. Nevertheless, when an extra structure is imposed to the RS's new difficulties may arise; such is the case of projective RS's (i.e. our analogue of fusion frames), where there are orthogonal relations between subsets of vectors, or injective RS's. Indeed, as far as we know Theorem 7 is new even in the vector frame setting. In addition, by the nature of its formulation, the approximation by projective RS's and the existence of projective dual RS's of a fixed projective RS are intrinsic problems to the RS's setting.

The paper is organized as follows: In Section 2 we recall some basic facts about general reconstruction systems and we fix some of the terminology used throughout the paper. In Section 3 we study the optimal dual systems for erasures of vector coefficients. In Section 4 we consider the problem of perfect reconstruction when packets corresponding to a fixed set of indices of a $\mathrm{RS} \mathcal{V}$ are lost. In Section 5 we describe the projective $\mathrm{RS}$ which is nearest to a fixed RS. In Section [6 we give several examples of group RS's, and we consider the problems of existence of projective dual RS's of a given projective RS.

\section{Notations.}

Given $\mathcal{H} \cong \mathbb{C}^{d}$ and $\mathcal{K} \cong \mathbb{C}^{n}$, we denote by $L(\mathcal{H}, \mathcal{K})$ the space of linear operators $T: \mathcal{H} \rightarrow \mathcal{K}$. Given an operator $T \in L(\mathcal{H}, \mathcal{K}), R(T) \subseteq \mathcal{K}$ denotes the image of $T$, $\operatorname{ker} T \subseteq \mathcal{H}$ the null space of $T$ and $T^{*} \in L(\mathcal{K}, \mathcal{H})$ the adjoint of $T$. If $d \leq n$ we say that $U \in L(\mathcal{H}, \mathcal{K})$ is an isometry if $U^{*} U=I_{\mathcal{H}}$. In this case, $U^{*}$ is called a coisometry. We denote by $\mathcal{I}(d, n)$ the set of all isometries in $L(\mathcal{H}, \mathcal{K})$.

If $\mathcal{K}=\mathcal{H}$ we denote by $L(\mathcal{H})=L(\mathcal{H}, \mathcal{H})$, by $G l(\mathcal{H})$ the group of all invertible operators in $L(\mathcal{H})$, by $L(\mathcal{H})^{+}$the cone of positive operators and by $G l(\mathcal{H})^{+}=G l(\mathcal{H}) \cap L(\mathcal{H})^{+}$.

If $T \in L(\mathcal{H})$, we denote by $\sigma(T)$ the spectrum of $T$, by $\operatorname{rk} T$ the $\operatorname{rank}$ of $T$, and by $\operatorname{tr} T$ the trace of $T$. Given $m \in \mathbb{N}$ we denote by $\mathbb{I}_{m}=\{1, \ldots, m\} \subseteq \mathbb{N}$ and $\mathbb{1}=\mathbb{1}_{m} \in \mathbb{R}^{m}$ denotes the vector with all its entries equal to 1.

On the other hand, $\mathcal{M}_{n, m}(\mathbb{C})$ denotes the space of complex $n \times m$ matrices. If $n=m$ we write $\mathcal{M}_{n}(\mathbb{C})=\mathcal{M}_{n, n}(\mathbb{C}), \mathcal{G} l(n)$ the group of all invertible elements of $\mathcal{M}_{n}(\mathbb{C}), \mathcal{U}(n)$ the group of unitary matrices, $\mathcal{M}_{n}(\mathbb{C})^{+}$the set of positive semidefinite matrices, and $\mathcal{G} l(n)^{+}=$ $\mathcal{M}_{n}(\mathbb{C})^{+} \cap \mathcal{G} l(n)$.

If $W \subseteq \mathcal{H}$ is a subspace we denote by $P_{W} \in L(\mathcal{H})^{+}$the orthogonal projection onto $W$, i.e. $R\left(P_{W}\right)=W$ and ker $P_{W}=W^{\perp}$. For vectors on $\mathbb{C}^{n}$ we shall use the euclidean norm, but for matrices $T \in \mathcal{M}_{n}(\mathbb{C})$, we shall use both

1. The spectral norm $\|T\|=\|T\|_{s p}=\max _{\|x\|=1}\|T x\|$.

2. The Frobenius norm $\|T\|_{2}=\left(\operatorname{tr} T^{*} T\right)^{1 / 2}=\left(\sum_{i, j \in \mathbb{I}_{n}}\left|T_{i j}\right|^{2}\right)^{1 / 2}$. This norm is induced by the inner product $\langle A, B\rangle=\operatorname{tr} B^{*} A$, for $A, B \in \mathcal{M}_{n}(\mathbb{C})$. 


\section{Basic framework of reconstruction systems}

In what follows we consider $(m, \mathbf{k}, d)$-reconstruction systems (see for example [15, 18, 20]), which are more general linear systems than those considered in [3, 4, 5, 6, 11] and [16], that also have an associated reconstruction algorithm.

Definition 1 Let $m, d \in \mathbb{N}$ and $\mathbf{k}=\left(k_{1}, \ldots, k_{m}\right) \in \mathbb{N}^{m}$.

1. We denote by $\mathcal{K}=\mathcal{K}_{m, \mathbf{k}} \stackrel{\text { def }}{=} \bigoplus_{i \in \mathbb{I}_{m}} \mathbb{C}^{k_{i}}$. Sometimes we shall write each direct summand by $\mathcal{K}_{i}=\mathbb{C}^{k_{i}}$.

2. Given a space $\mathcal{H} \cong \mathbb{C}^{d}$ we denote by $L(m, \mathbf{k}, d) \stackrel{\text { def }}{=} \bigoplus_{i \in \mathbb{I}_{m}} L\left(\mathcal{H}, \mathcal{K}_{i}\right) \cong L(\mathcal{H}, \mathcal{K})$. A typical element of $L(m, \mathbf{k}, d)$ is a system $\mathcal{V}=\left\{V_{i}\right\}_{i \in \mathbb{I}_{m}}$ such that each $V_{i} \in L\left(\mathcal{H}, \mathcal{K}_{i}\right)$.

3. A family $\mathcal{V}=\left\{V_{i}\right\}_{i \in \mathbb{I}_{m}} \in L(m, \mathbf{k}, d)$ is an $(m, \mathbf{k}, d)$-reconstruction system (RS) for $\mathcal{H}$ if

$$
S_{\mathcal{V}} \stackrel{\text { def }}{=} \sum_{i \in \mathbb{I}_{m}} V_{i}^{*} V_{i} \in G l(\mathcal{H})^{+}
$$

i.e., if $S_{\mathcal{V}}$ is invertible and positive. $S_{\mathcal{V}}$ is called the $\mathbf{R S}$ operator of $\mathcal{V}$. In this case, the $m$-tuple $\mathbf{k}=\left(k_{1}, \ldots, k_{m}\right) \in \mathbb{N}^{m}$ satisfies that $\operatorname{tr} \mathbf{k} \stackrel{\text { def }}{=} \sum_{i \in \mathbb{I}_{m}} k_{i} \geq d$.

4. The bounds of $\mathcal{V}$ as a RS are the numbers $A_{\mathcal{V}}=\lambda_{\min }\left(S_{\mathcal{V}}\right)$ and $B_{\mathcal{V}}=\left\|S_{\mathcal{V}}\right\|_{s p}$. Observe that

$$
A_{\mathcal{V}}\|x\|^{2} \leq\left\langle S_{\mathcal{V}} x, x\right\rangle=\sum_{i \in \mathbb{I}_{m}}\left\|V_{i} x\right\|^{2} \leq B_{\mathcal{V}}\|x\|^{2} \quad \text { for every } \quad x \in \mathcal{H}
$$

5. We shall denote by $\mathcal{R S}(m, \mathbf{k}, d)$ the set of all $(m, \mathbf{k}, d)$-RS's for $\mathcal{H} \cong \mathbb{C}^{d}$.

6. The system $\mathcal{V}=\left\{V_{i}\right\}_{i \in \mathbb{I}_{m}} \in \mathcal{R} \mathcal{S}(m, \mathbf{k}, d)$ is said to be injective if $V_{i}^{*} \in L\left(\mathcal{K}_{i}, \mathcal{H}\right)$ is injective (equivalently, if $V_{i} V_{i}^{*} \in G l\left(\mathcal{K}_{i}\right)$ ) for every $i \in \mathbb{I}_{m}$.

We shall denote by $\mathcal{R S}_{\mathcal{I}}(m, \mathbf{k}, d)$ the set of all injective elements of $\mathcal{R} \mathcal{S}(m, \mathbf{k}, d)$.

7. The system $\mathcal{V}$ is said to be projective if there exists a sequence $\mathbf{v}=\left(v_{i}\right)_{i \in \mathbb{I}_{m}} \in \mathbb{R}_{+}^{m}$ of positive numbers, the weights of $\mathcal{V}$, such that

$$
V_{i} V_{i}^{*}=v_{i}^{2} I_{\mathcal{K}_{i}}, \quad \text { for every } \quad i \in \mathbb{I}_{m}
$$

In this case, the following properties hold:

(a) The weights can be computed directly, since each $v_{i}=\left\|V_{i}\right\|_{s p}$.

(b) Each $V_{i}=v_{i} U_{i}$ for a coisometry $U_{i} \in L\left(\mathcal{H}, \mathcal{K}_{i}\right)$. Thus $V_{i}^{*} V_{i}=v_{i}^{2} P_{R\left(V_{i}^{*}\right)} \in L(\mathcal{H})^{+}$ for every $i \in \mathbb{I}_{m}$.

(c) Observe that in this case $S_{\mathcal{V}}=\sum_{i \in \mathbb{I}_{m}} v_{i}^{2} P_{R\left(V_{i}^{*}\right)}$ as in fusion frame theory.

We shall denote by $\mathcal{P}(m, \mathbf{k}, d)$ the set of all projective elements of $\mathcal{R} \mathcal{S}(m, \mathbf{k}, d)$.

8. The analysis operator of the system $\mathcal{V}$ is defined by

$$
T_{\mathcal{V}}: \mathcal{H} \rightarrow \mathcal{K}=\bigoplus_{i \in \mathbb{I}_{m}} \mathcal{K}_{i} \text { given by } T_{\mathcal{V}} x=\left(V_{1} x, \ldots, V_{m} x\right), \quad \text { for } \quad x \in \mathcal{H}
$$


9. Its adjoint $T_{\mathcal{V}}^{*}$ is called the synthesis operator of the system $\mathcal{V}$, and it satisfies that

$$
T_{\mathcal{V}}^{*}: \mathcal{K}=\bigoplus_{i \in \mathbb{I}_{m}} \mathcal{K}_{i} \rightarrow \mathcal{H} \quad \text { is given by } \quad T_{\mathcal{V}}^{*}\left(\left(y_{i}\right)_{i \in \mathbb{I}_{m}}\right)=\sum_{i \in \mathbb{I}_{m}} V_{i}^{*} y_{i}
$$

Using the previous notations and definitions we have that $S_{\mathcal{V}}=T_{\mathcal{V}}^{*} T_{\mathcal{V}}$.

\section{Examples 2 (Vector and fusion frames as RS's)}

1. As it was mentioned earlier, RS's arise from usual vector frames by grouping together the elements of the frame. Therefore, it is natural to expect that in the case $\mathbf{k}=\mathbb{1}_{m}$, the set $\mathcal{R S}(m, \mathbf{k}, d)$ can be identified with the set of $m$-vector frames for $\mathcal{H} \cong \mathbb{C}^{d}$.

Indeed, let $\mathcal{F}=\left\{f_{i}\right\}_{i \in \mathbb{I}_{m}} \in \mathcal{H}^{m}$. For $i \in \mathbb{I}_{m}$ consider $V_{i}: \mathcal{H} \rightarrow \mathbb{C}$ given by $V_{i}(x)=\left\langle x, f_{i}\right\rangle$ for every $x \in \mathcal{H}$. Let $\mathcal{V}_{\mathcal{F}}=\left\{V_{i}\right\}_{i \in \mathbb{I}_{m}}$ and notice that

$$
S_{\mathcal{V}_{\mathcal{F}}}=\sum_{i \in \mathbb{I}_{m}} V_{i}^{*} V_{i}=\sum_{i \in \mathbb{I}_{m}}\left\langle\cdot, f_{i}\right\rangle f_{i}=S_{\mathcal{F}}
$$

Thus $\mathcal{F}$ is a frame for $\mathcal{H}$ if and only if $\mathcal{V}_{\mathcal{F}} \in \mathcal{R} \mathcal{S}(m, \mathbb{1}, d)$. Actually, $\mathcal{R} \mathcal{S}(m, \mathbb{1}, d)=\mathcal{P}(m, \mathbb{1}, d)$ because every functional is a multiple of a coisometry. Moreover, $T_{\mathcal{V}_{\mathcal{F}}}: \mathcal{H} \rightarrow \oplus_{i \in \mathbb{I}_{m}} \mathbb{C}=\mathbb{C}^{m}$ is the usual analysis operator of $\mathcal{F}$. On the other hand, it is clear that elements in $\mathcal{R} \mathcal{S}(m, \mathbb{1}, d)$ correspond to vector frames for $\mathcal{H}$.

2. Let $\mathcal{N}_{w}=\left(w_{i}, \mathcal{N}_{i}\right)_{i \in \mathbb{I}_{m}}$ be a fusion frame for $\mathcal{H} \cong \mathbb{C}^{d}$, with weights $w_{i}>0$ and subspaces $\mathcal{N}_{i} \subseteq \mathcal{H}$ with $\operatorname{dim} \mathcal{N}_{i}=k_{i}$ for every $i \in \mathbb{I}_{m}$. Its fusion frame operator is

$$
S_{\mathcal{N}_{w}}=\sum_{i \in \mathbb{I}_{m}} w_{i}^{2} P_{\mathcal{N}_{i}} \in G l(\mathcal{H})^{+}
$$

(see [8, 9, 12] for detailed expositions of fusion frames). Let $U_{i} \in L\left(\mathcal{H}, \mathbb{C}^{k_{i}}\right)$ be a coisometry such that $U_{i}^{*} U_{i}=P_{\mathcal{N}_{i}}$, for every $i \in \mathbb{I}_{m}$. Therefore, the system $\mathcal{V}=\left\{V_{i}\right\}_{i \in \mathbb{I}_{m}} \stackrel{\text { def }}{=}\left\{w_{i} U_{i}\right\}_{i \in \mathbb{I}_{m}}$ satisfies that $S_{\mathcal{V}}=S_{\mathcal{N}_{w}} \in G l(\mathcal{H})^{+}$. Hence $\mathcal{V} \in \mathcal{P}(m, \mathbf{k}, d)$ is a projective RS associated to $\mathcal{N}_{w}$. Observe that $\mathcal{V}$ has the same weights as $\mathcal{N}_{w}$ and it also satisfies that each $\mathcal{N}_{i}=R\left(V_{i}^{*}\right)$.

Conversely, given $\mathcal{V}=\left\{V_{i}\right\}_{i \in \mathbb{I}_{m}} \in \mathcal{P}(m, \mathbf{k}, d)$, the sequence $\mathcal{N}_{w}=\left(\left\|V_{i}\right\|, R\left(V_{i}^{*}\right)\right)_{i \in \mathbb{I}_{m}}$ is a fusion frame such that $S_{\mathcal{V}}=S_{\mathcal{N}_{w}}$. Nevertheless the correspondence is not one to one, since any system of coisometries $\left\{U_{i}\right\}_{i \in \mathbb{I}_{m}}$ with $\left(\operatorname{ker} U_{i}\right)^{\perp}=\mathcal{N}_{i}$ produces the same fusion frame $\mathcal{N}_{w}=\left(w_{i}, \mathcal{N}_{i}\right)_{i \in \mathbb{I}_{m}}$. This phenomenon is similar to the correspondence of vector frames with one dimensional fusion frames.

Remark 3 In what follows we list some properties and notations about RS's:

1. Given $\mathcal{V}=\left\{V_{i}\right\}_{i \in \mathbb{I}_{m}} \in \mathcal{R} \mathcal{S}(m, \mathbf{k}, d)$ with $S_{\mathcal{V}}=\sum_{i \in \mathbb{I}_{m}} V_{i}^{*} V_{i}$, then

$$
\sum_{i \in \mathbb{I}_{m}} S_{\mathcal{V}}{ }^{-1} V_{i}^{*} V_{i}=I_{\mathcal{H}}, \quad \text { and } \quad \sum_{i \in \mathbb{I}_{m}} V_{i}^{*} V_{i} S_{\mathcal{V}}{ }^{-1}=I_{\mathcal{H}}
$$

Therefore, we obtain the reconstruction formulas

$$
x=\sum_{i \in \mathbb{I}_{m}} S_{\mathcal{V}}^{-1} V_{i}^{*}\left(V_{i} x\right)=\sum_{i \in \mathbb{I}_{m}} V_{i}^{*} V_{i}\left(S_{\mathcal{V}}^{-1} x\right) \quad \text { for every } \quad x \in \mathcal{H} .
$$


2. For every $\mathcal{V}=\left\{V_{i}\right\}_{i \in \mathbb{I}_{m}} \in \mathcal{R} \mathcal{S}(m, \mathbf{k}, d)$, we define the system

$$
\mathcal{V}^{\#} \stackrel{\text { def }}{=}\left\{V_{i} S_{\mathcal{V}}^{-1}\right\}_{i \in \mathbb{I}_{m}} \in \mathcal{R} \mathcal{S}(m, \mathbf{k}, d),
$$

called the canonical dual RS associated to $\mathcal{V}$. By Eq. (11), we see that

$$
T_{\mathcal{V} \#}^{*} T_{\mathcal{V}}=\sum_{i \in \mathbb{I}_{m}} S_{\mathcal{V}}{ }^{-1} V_{i}^{*} V_{i}=I_{\mathcal{H}} \quad \text { and } \quad S_{\mathcal{V} \#}=\sum_{i \in \mathbb{I}_{m}} S_{\mathcal{V}}^{-1} V_{i}^{*} V_{i} S_{\mathcal{V}}^{-1}=S_{\mathcal{V}}^{-1}
$$

Next we generalize the notion of dual RS's :

Definition 4 Let $\mathcal{V}=\left\{V_{i}\right\}_{i \in \mathbb{I}_{m}}$ and $\mathcal{W}=\left\{W_{i}\right\}_{i \in \mathbb{I}_{m}} \in \mathcal{R} \mathcal{S}(m, \mathbf{k}, d)$.

1. We say that $\mathcal{W}$ is a dual RS for $\mathcal{V}$ if $T_{\mathcal{W}}^{*} T_{\mathcal{V}}=I_{\mathcal{H}}$, or equivalently if

$$
x=\sum_{i \in \mathbb{I}_{m}} W_{i}^{*} V_{i} x \quad \text { for every } \quad x \in \mathcal{H} .
$$

2. We denote by $D(\mathcal{V}) \stackrel{\text { def }}{=}\left\{\mathcal{W} \in \mathcal{R S}(m, \mathbf{k}, d): T_{\mathcal{W}}^{*} T_{\mathcal{V}}=I_{\mathcal{H}}\right\}$, the set of all dual RS's for a fixed $\mathcal{V} \in \mathcal{R} \mathcal{S}(m, \mathbf{k}, d)$. Observe that $D(\mathcal{V}) \neq \varnothing$ since $\mathcal{V}^{\#} \in D(\mathcal{V})$.

Remark 5 Let $\mathcal{V} \in \mathcal{R} \mathcal{S}(m, \mathbf{k}, d)$. Then $\mathcal{W} \in D(\mathcal{V})$ if and only if its synthesis operator $T_{\mathcal{W}}^{*}$ is a pseudo-inverse of $T_{\mathcal{V}}$. Indeed, $\mathcal{W} \in D(\mathcal{V}) \Longleftrightarrow T_{\mathcal{W}}^{*} T_{\mathcal{V}}=I_{\mathcal{H}}$. Observe that the map $\mathcal{R S}(m, \mathbf{k}, d) \ni \mathcal{W} \mapsto T_{\mathcal{W}}^{*}$ is one to one. Thus, in the context of RS's each $(m, \mathbf{k}, d)$-RS has many duals that are $(m, \mathbf{k}, d)$-RS's. This is one of the advantages of the RS's setting.

Moreover, the synthesis operator $T_{\mathcal{V} \#}^{*}$ of the canonical dual $\mathcal{V}^{\#}$ corresponds to the MoorePenrose pseudo-inverse of $T_{\mathcal{V}}$. Indeed, notice that $T_{\mathcal{V}} T_{\mathcal{V} \#}^{*}=T_{\mathcal{V}} S_{\mathcal{V}}^{-1} T_{\mathcal{V}}^{*} \in L(\mathcal{K})^{+}$, so that it is an orthogonal projection. From this point of view, $\mathcal{V}^{\#}$ has some optimal properties that come from the theory of pseudo-inverses.

\section{Optimal erasures and errors}

In coding theory, and using our terminology, a signal $x \in \mathbb{C}^{d}$ is transmitted encoded via the RS $\mathcal{V}=\left\{V_{i}\right\}_{i \in \mathbb{I}_{m}}$ in the form of $m$ packets $\left\{V_{i} x\right\}_{i \in \mathbb{I}_{m}}$. Then, the loss (or delay) of a number of packets in transmission is treated as if the corresponding components in the RS were "erased". Assuming that a number of erasures occurred and taking into account the redundancy of the RS, the reconstruction of the signal could be attempted using a dual RS of $\mathcal{V}$. The accuracy of this process can be quantified by the norm of an error operator. For example, it is shown in [11] that uniform Parseval frames (together with their canonical duals) are optimal for 1 erasures in the sense that they minimize a norm of the error operator. The authors also prove that equiangular uniform Parseval frames are optimal for 2 erasures (in case that such frames exist). In the context of Parseval fusion frames, Bodmann ([4) shows that uniform Parseval fusion frames (a protocol associated to a uniformly weighted projective resolution of the identity, using his terminology) are optimal for 1 erasure, when the dimensions of the subspaces are equal. In addition, he shows that the uniform Parseval protocols that are optimal for multiple erasures have subspaces satisfying the so-called equiisoclinic condition. 
Similar problems have also been considered in different contexts. For example, in [12] the authors describe the structure of the optimal frames for one or multiple erasures, but in a non-deterministic setting. Indeed, in this case a random vector $x$ is estimated from its fusion frame measurements using the Linear Minimum Mean Square Error.

As it was mentioned in the introduction, our aim is to set the problem studied in [14] in the context of reconstruction systems. That is, given a fixed projective $\mathrm{RS} \mathcal{V}$, the goal is to characterize optimal alternate duals for $r$ erasures. As in [14], our results imply the existence of a unique dual RS (which is not necessarily projective) that is optimal for the erasure of 1 packet of coefficients. Notice that in this context, optimal alternate duals for $r$ erasures among those duals which are optimal for $r-1$ erasures (see [4, 5, 6, 11, 14, 15]). Therefore, by the nature of our results, the optimal duals for $r$ erasures coincide with optimal duals for 1 erasure.

In order to describe the different measures of the reconstruction error when an arbitrary packet of coefficients of the fixed RS is erased, we consider the following notions. Given $j \in \mathbb{I}_{m}$, let

$$
M_{j} \in L(\mathcal{K}) \quad \text { given by } \quad M_{j}\left(\left(y_{i}\right)_{i \in \mathbb{I}_{m}}\right)=\left(\mathbb{1}_{j}(i) \cdot y_{i}\right)_{i \in \mathbb{I}_{m}},
$$

where $\mathbb{1}_{j}: \mathbb{I}_{m} \rightarrow\{0,1\}$ denotes the characteristic function of the set $\{j\} \subset \mathbb{I}_{m}$. Similarly, we consider the packet-lost operator $L_{j} \stackrel{\text { def }}{=} M_{\mathbb{I}_{m} \backslash\{j\}}=I_{\mathcal{K}}-M_{j}$.

Given $\mathcal{V}=\left\{V_{i}\right\}_{i \in \mathbb{I}_{m}} \in \mathcal{R S}(m, \mathbf{k}, d)$, we shall consider a "blind reconstruction" strategy in case that some coefficient is lost. That is, assuming that the encoded information $T_{\mathcal{V}} x \in \mathcal{K}$ (for some $x \in \mathcal{H}$ ) is altered according to the packet-lost operator $L_{j}$, our reconstructed vector will be $\hat{x}=T_{\mathcal{W}}^{*} L_{j} T_{\mathcal{V}}(x)$, where $\mathcal{W}=\left\{W_{i}\right\}_{i \in \mathbb{I}_{m}} \in D(\mathcal{V})$ is some dual RS for $\mathcal{V}$. Then the reconstruction error will be $x-\hat{x}=x-T_{\mathcal{W}}^{*} L_{j} T_{\mathcal{V}}(x)=T_{\mathcal{W}}^{*} M_{j} T_{\mathcal{V}}(x)=W_{j}^{*} V_{j} x$.

In this case, we will use the Frobenius norm $\|\cdot\|_{2}$ to perform the measure of the operator $W_{j}^{*} V_{j}$. It is worth to note that, unlike the vector frame case, $W_{j}^{*} V_{j}$ is not necessary a rankone operator, so its operator (spectral) norm does not coincide with its Frobenius norm. We consider that this is a suitable norm to perform the generalization of the results in [14] to the RS setting, besides the fact that Frobenius norm has nice geometrical properties. Consider the $m$-tuple

$$
E_{1}(\mathcal{V}, \mathcal{W})=\left(\left\|I-T_{\mathcal{W}}^{*} L_{j} T_{\mathcal{V}}\right\|_{2}\right)_{j \in \mathbb{I}_{m}}=\left(\left\|T_{\mathcal{W}}^{*} M_{j} T_{\mathcal{V}}\right\|_{2}\right)_{j \in \mathbb{I}_{m}}=\left(\left\|W_{j}^{*} V_{j}\right\|_{2}\right)_{j \in \mathbb{I}_{m}} \in \mathbb{R}^{m},
$$

Notice that we can bound uniformly the reconstruction error in terms of the entries of this vector for the erasure of 1 packet of coefficients (for all $m$ possible choices). In what follows we shall consider two different measures of the reconstruction error based on $E_{1}(\mathcal{V}, \mathcal{W})$, namely the 2-error and the (normalized) worst-case error.

\subsection{Minimizing the 2-error}

Let $\mathcal{V}=\left\{V_{i}\right\}_{i \in \mathbb{I}_{m}} \in \mathcal{R} \mathcal{S}(m, \mathbf{k}, d)$ and let us denote by

$$
e_{1}^{(2)}(\mathcal{V})=\inf _{\mathcal{W} \in D(\mathcal{V})}\left\|E_{1}(\mathcal{V}, \mathcal{W})\right\|_{2}=\inf _{\mathcal{W} \in D(\mathcal{V})}\left(\sum_{i \in \mathbb{I}_{m}}\left\|W_{i}^{*} V_{i}\right\|_{2}^{2}\right)^{1 / 2} .
$$

We are interested in the characterization of those $\mathcal{W} \in D(\mathcal{V})$ such that $\left\|E_{1}(\mathcal{V}, \mathcal{W})\right\|_{2}=$ $e_{1}^{(2)}(\mathcal{V})$. In other words, we define the set of 1-loss optimal dual RS's for $\mathcal{V}$ with respect to $\left\|E_{1}(\mathcal{V}, \cdot)\right\|_{2}$ as

$$
D_{1}^{(2)}(\mathcal{V}) \stackrel{\text { def }}{=}\left\{\mathcal{W} \in D(\mathcal{V}):\left\|E_{1}(\mathcal{V}, \mathcal{W})\right\|_{2}=e_{1}^{(2)}(\mathcal{V})\right\}
$$


One of the advantages of this measure of the error is that for a projective $\mathcal{V}$, without any further assumption, there is always only one dual RS in $D_{1}^{(2)}(\mathcal{V})$, and that this system can be explicitely computed (see Theorem 7 and Remark 8). In particular, using the hierarchies previously described, this dual will also be optimal for $r$ erasures.

Remark 6 Fix $\mathcal{V}=\left\{V_{i}\right\}_{i \in \mathbb{I}_{m}} \in \mathcal{P}(m, \mathbf{k}, d)$ and $\mathcal{W}=\left\{W_{i}\right\}_{i \in \mathbb{I}_{m}} \in D(\mathcal{V})$. We give a matricial interpretation of the 2-error $\left\|E_{1}(\mathcal{V}, \mathcal{W})\right\|_{2}$, for the sake of clarity. Recall that $\mathcal{V}, \mathcal{W} \in$ $L(m, \mathbf{k}, d)=\bigoplus_{i \in \mathbb{I}_{m}} L\left(\mathcal{H}, \mathcal{K}_{i}\right)$. Hence they can be viewed as block $m \times 1$ column vectors. For example, this allows to obtain the equality $I=T_{\mathcal{W}}^{*} T_{\mathcal{V}}=\sum_{i \in \mathbb{I}_{m}} W_{i}^{*} V_{i}$; unfortunately this identity does not allow us to compute the value of $\left\|E_{1}(\mathcal{V}, \mathcal{W})\right\|_{2}$.

On the other hand, the product $T_{\mathcal{V}} T_{\mathcal{W}}^{*}=\sum_{i, j \in \mathbb{I}_{m}} V_{i} W_{j}^{*} \in L(\mathcal{K})$ is an oblique projector, and $\left\|T_{\mathcal{V}} T_{\mathcal{W}}^{*}\right\|_{2}^{2}=\sum_{i, j \in \mathbb{I}_{m}}\left\|V_{i} W_{j}^{*}\right\|_{2}^{2}=\sum_{i, j \in \mathbb{I}_{m}}\left\|W_{j}^{*} V_{i}\right\|_{2}^{2}$, since the blocks $V_{i} W_{j}^{*} \in L\left(\mathcal{K}_{j}, \mathcal{K}_{i}\right)$ are the different "entries" of the $m \times m$ block matrix $T_{\mathcal{V}} T_{\mathcal{W}}^{*}$.

The norm $\left\|T_{\mathcal{V}} T_{\mathcal{W}}^{*}\right\|_{2}$ is minimal for $\mathcal{W}=\mathcal{V}^{\#}$ (since $T_{\mathcal{V}} T_{\mathcal{V}}^{*}$ is selfadjoint). Nevertheless, the square of the 2-error $\left\|E_{1}(\mathcal{V}, \mathcal{W})\right\|_{2}^{2}=\sum_{i \in \mathbb{I}_{m}}\left\|W_{i}^{*} V_{i}\right\|_{2}^{2}$ is the square of the norm of the pinching matrix (i.e., block diagonal truncation) of $T_{\mathcal{V}} T_{\mathcal{W}}^{*}$. As we shall see below, its minimum among all $\mathcal{W} \in D(\mathcal{V})$ is not always attained at the Moore Penrose pseudoinverse $T_{\mathcal{V} \#}^{*}($ see Remark 10).

Theorem 7 Let $\mathcal{V}=\left\{V_{i}\right\}_{i \in \mathbb{I}_{m}} \in \mathcal{P}(m, \mathbf{k}, d)$ with weights $\mathbf{v}=\left(v_{i}\right)_{i \in \mathbb{I}_{m}}$. Then $D_{1}^{(2)}(\mathcal{V})=$ $\left\{\mathcal{W}_{0}\right\}$ i.e., there is a unique 1-loss optimal dual $R S \mathcal{W}_{0}$ for the 2-error. Moreover, if

1. $D \in L(\mathcal{K})$ is the block diagonal matrix $D=\bigoplus_{i \in \mathbb{I}_{m}} v_{i}^{-2} I_{\mathcal{K}_{i}}$, and

2. $S_{\mathcal{V}, D}=T_{\mathcal{V}}^{*} D T_{\mathcal{V}}=\sum_{i \in \mathbb{I}_{m}} P_{R\left(V_{i}^{*}\right)} \in G l(\mathcal{H})^{+}\left(\operatorname{since} S_{\mathcal{V}, D} \geq\left(\min _{i \in \mathbb{I}_{m}} v_{i}^{-2}\right) \cdot S_{\mathcal{V}}>0\right)$,

then the optimal system is $\mathcal{W}_{0}=\left\{v_{i}^{-2} V_{i} S_{\mathcal{V}, D}^{-1}\right\}_{i \in \mathbb{I}_{m}}$. In particular, $T_{\mathcal{W}_{0}}=D T_{\mathcal{V}} S_{\mathcal{V}, D}^{-1}$.

Proof. First we check that $\mathcal{W}_{0} \in D(\mathcal{V})$. Indeed, $T_{\mathcal{W}_{0}}^{*} T_{\mathcal{V}}=S_{\mathcal{V}, D}^{-1} T_{\mathcal{V}}^{*} D T_{\mathcal{V}}=S_{\mathcal{V}, D}^{-1} S_{\mathcal{V}, D}=I$. Denote by $B_{i}=v_{i}^{-2} V_{i} S_{\mathcal{V}, D}^{-1}$, the $i$-th entry of $\mathcal{W}_{0}$, for every $i \in \mathbb{I}_{m}$. Consider a dual system $\mathcal{W}=\left\{W_{i}\right\}_{i \in \mathbb{I}_{m}} \in D(\mathcal{V})$. Since each $V_{i} V_{i}^{*}=v_{i}^{2} I_{\mathcal{K}_{i}}$, then

$$
\left\|W_{i}^{*} V_{i}\right\|_{2}^{2}=\operatorname{tr}\left(V_{i}^{*} W_{i} W_{i}^{*} V_{i}\right)=\operatorname{tr}\left(W_{i} W_{i}^{*} V_{i} V_{i}^{*}\right)=v_{i}^{2} \operatorname{tr}\left(W_{i} W_{i}^{*}\right)=v_{i}^{2}\left\|W_{i}^{*}\right\|_{2}^{2} .
$$

In particular, we can compute the 2-error for $\mathcal{W}_{0}$ :

$$
\left\|E_{1}\left(\mathcal{V}, \mathcal{W}_{0}\right)\right\|^{2}=\sum_{i \in \mathbb{I}_{m}}\left\|B_{i}^{*} V_{i}\right\|_{2}^{2} \stackrel{(\underline{3})}{=} \sum_{i \in \mathbb{I}_{m}} v_{i}^{2}\left\|B_{i}^{*}\right\|_{2}^{2}=\sum_{i \in \mathbb{I}_{m}} v_{i}^{-2}\left\|S_{\mathcal{V}, D}^{-1} V_{i}^{*}\right\|_{2}^{2} .
$$

On the other hand, for every $i \in \mathbb{I}_{m}$ we have

$$
\begin{aligned}
v_{i}^{2}\left\|W_{i}^{*}\right\|_{2}^{2} & =v_{i}^{2}\left\|B_{i}^{*}+\left(W_{i}^{*}-B_{i}^{*}\right)\right\|_{2}^{2} \\
& =v_{i}^{-2}\left\|S_{\mathcal{V}, D}^{-1} V_{i}^{*}\right\|_{2}^{2}+v_{i}^{2}\left\|W_{i}^{*}-B_{i}^{*}\right\|_{2}^{2}+2 \operatorname{Re}\left(v_{i}^{2} \operatorname{tr}\left[\left(W_{i}^{*}-B_{i}^{*}\right) B_{i}\right]\right) .
\end{aligned}
$$

Let $t_{i}=\operatorname{tr}\left[\left(W_{i}^{*}-B_{i}^{*}\right) B_{i}\right]=v_{i}^{-2} \operatorname{tr}\left[\left(W_{i}^{*}-B_{i}^{*}\right) V_{i} S_{\mathcal{V}, D}^{-1}\right]$. Then we have that

$$
\sum_{i \in \mathbb{I}_{m}} v_{i}^{2} t_{i}=\operatorname{tr}\left(\sum_{i \in \mathbb{I}_{m}}\left(W_{i}^{*}-B_{i}^{*}\right) V_{i} S_{\mathcal{V}, D}^{-1}\right)=\operatorname{tr}\left[\left(T_{\mathcal{W}}^{*}-T_{\mathcal{W}_{0}}^{*}\right) T_{\mathcal{V}} S_{\mathcal{V}, D}^{-1}\right]=0
$$


since both $\mathcal{W}$ and $\mathcal{V}^{\#}$ are dual RS's for $\mathcal{V}$. Therefore, summing over $\mathbb{I}_{m}$, the third summand of (41) vanishes and

$$
\begin{aligned}
\left\|E_{1}(\mathcal{V}, \mathcal{W})\right\|^{2}=\sum_{i \in \mathbb{I}_{m}}\left\|W_{i}^{*} V_{i}\right\|_{2}^{2} & \stackrel{(3)}{=} \sum_{i \in \mathbb{I}_{m}} v_{i}^{2}\left\|W_{i}^{*}\right\|_{2}^{2} \\
& \stackrel{(4)}{=} \sum_{i \in \mathbb{I}_{m}} v_{i}^{-2}\left\|S_{\mathcal{V}, D}^{-1} V_{i}^{*}\right\|_{2}^{2}+v_{i}^{2}\left\|W_{i}^{*}-B_{i}^{*}\right\|_{2}^{2} \\
& \geq \sum_{i \in \mathbb{I}_{m}} v_{i}^{2}\left\|B_{i}^{*}\right\|_{2}^{2} \stackrel{(\underline{3})}{=}\left\|E_{1}\left(\mathcal{V}, \mathcal{W}_{0}\right)\right\|^{2} .
\end{aligned}
$$

Therefore $\mathcal{W}_{0} \in D_{1}^{(2)}(\mathcal{V})$. Moreover, if we take another $\mathcal{W}=\left\{W_{i}\right\}_{i \in \mathbb{I}_{m}} \in D_{1}^{(2)}(\mathcal{V})$, then Eq. (5) implies that $\left\|W_{i}^{*}-B_{i}^{*}\right\|_{2}=0$ for every $i \in \mathbb{I}_{m}$, so that $\mathcal{W}=\mathcal{W}_{0}$.

We say that a system $\mathcal{V}=\left\{V_{i}\right\}_{i \in \mathbb{I}_{m}} \in \mathcal{P}(m, \mathbf{k}, d)$ is an uniform projective RS if the weights $\mathbf{v}=\left(v_{i}\right)_{i \in \mathbb{I}_{m}}$ of $\mathcal{V}$ satisfy that $\mathbf{v}=v \mathbb{1}$ for some $v>0$.

Remark 8 The unique 1-loss optimal dual RS of Theorem 7 , denoted by $\mathcal{W}_{0} \in D_{1}^{(2)}(\mathcal{V})$, can be described in the following way: If $\mathbf{v}=\left(v_{i}\right)_{i \in \mathbb{I}_{m}}$ are the weights of the system $\mathcal{V} \in$ $\mathcal{P}(m, \mathbf{k}, d)$, consider $\mathcal{U}=\left\{U_{i}\right\}_{i \in \mathbb{I}_{m}}=\left\{\frac{V_{i}}{v_{i}}\right\}_{i \in \mathbb{I}_{m}}$, which is a uniform projective RS. Then

$$
S_{\mathcal{V}, D}=\sum_{i \in \mathbb{I}_{m}} P_{R\left(U_{i}^{*}\right)}=S_{\mathcal{U}} \quad, \quad \mathcal{V}=\mathbf{v} \cdot \mathcal{U} \stackrel{\text { def }}{=}\left\{v_{i} U_{i}\right\}_{i \in \mathbb{I}_{m}} \quad \text { and } \quad \mathcal{W}_{0}=\mathbf{v}^{-1} \cdot \mathcal{U}^{\#}
$$

because $\mathcal{W}_{0}=\left\{v_{i}^{-2} V_{i} S_{\mathcal{V}, D}^{-1}\right\}_{i \in \mathbb{I}_{m}}=\left\{v_{i}^{-1} U_{i} S_{\mathcal{U}}^{-1}\right\}_{i \in \mathbb{I}_{m}}$.

Corollary 9 Let $\mathcal{V}=\left\{V_{i}\right\}_{i \in \mathbb{I}_{m}} \in \mathcal{P}(m, \mathbf{k}, d)$. Assume that $\mathcal{V}$ is uniform. Then the unique 1-loss optimal dual $R S$ for the 2-error is the canonical dual $\mathcal{V}^{\# .}$

Proof. If the weights of $\mathcal{V}$ are $\mathbf{v}=v \mathbb{1}$ then, with the notations of Remark 8 , we have that $\mathcal{V}=v \mathcal{U}, S_{\mathcal{V}}=v^{2} S_{\mathcal{U}}$ and $\mathcal{V}^{\#}=v^{-1} \mathcal{U}^{\#}$. Then we apply Eq. (6).

Remark 10 For most systems $\mathcal{V}=\left\{V_{i}\right\}_{i \in \mathbb{I}_{m}} \in \mathcal{P}(m, \mathbf{k}, d)$ which are not uniform, the unique 1-loss optimal dual RS for the 2-error $\mathcal{W}_{0} \in D(\mathcal{V})$ obtained in Theorem 7 does not coincide with the canonical dual $\mathcal{V}^{\#}$. For example, it is easy to see that $\mathcal{W}_{0} \neq \mathcal{V}^{\#}$ if all the weights are different and if the subspaces $R\left(V_{i}^{*}\right)$ are not mutually orthogonal. In particular, $\mathcal{W}_{0} \neq \mathcal{V}^{\#}$ whenever the weights are different and $\mathcal{V}$ has non zero redundancy.

\subsection{Minimizing the worst-case reconstruction error}

Let $\mathcal{V}=\left\{V_{i}\right\}_{i \in \mathbb{I}_{m}} \in \mathcal{P}(m, \mathbf{k}, d)$. For each $\mathcal{W}=\left\{W_{i}\right\}_{i \in \mathbb{I}_{m}} \in D(\mathcal{V})$, we introduce the worstcase reconstruction error when one packet is lost with respect to the Frobenius norm. Now, we measure the error vector $E_{1}(\mathcal{V}, \mathcal{W})$ with the maximum of its entries, instead of the euclidean norm used in the previous section.

$$
e_{1}(\mathcal{V})=\inf _{\mathcal{W} \in D(\mathcal{V})}\left\|E_{1}(\mathcal{V}, \mathcal{W})\right\|_{\infty}=\inf _{\mathcal{W} \in D(\mathcal{V})} \max _{i \in \mathbb{I}_{m}}\left\|T_{\mathcal{W}}^{*} M_{i} T_{\mathcal{V}}\right\|_{2}=\inf _{\mathcal{W} \in D(\mathcal{V})} \max _{i \in \mathbb{I}_{m}}\left\|W_{i}^{*} V_{i}\right\|_{2}
$$


We define the set of 1 -loss optimal dual RS's for $\mathcal{V}$ with respect to $\left\|E_{1}(\mathcal{V}, \cdot)\right\|_{\infty}$ as

$$
D_{1}(\mathcal{V}) \stackrel{\text { def }}{=}\left\{\mathcal{W} \in D(\mathcal{V}):\left\|E_{1}(\mathcal{V}, \mathcal{W})\right\|_{\infty}=e_{1}(\mathcal{V})\right\}
$$

The study of $D_{1}(\mathcal{V})$ has been considered by Han and López in [14] in the particular case of $(m, \mathbb{1}, d)$-RS's for $\mathcal{H}$, i.e. usual vector frames. Indeed, since in such case the operators $i \in \mathbb{I}_{m}, T_{\mathcal{W}}^{*} M_{i} T_{\mathcal{V}}$ are rank one operators, their Frobenius and spectral norms coincide, so the WCE coincide with the measure of the error used in [14]. The use of the Frobenius norm in the definition of the WCE allows to extend naturally the results in [14] to the RS setting using similar techniques.

Remark 11 Recall that $\mathcal{R S}(m, \mathbf{k}, d) \subseteq L(m, \mathbf{k}, d)=\bigoplus_{i \in \mathbb{I}_{m}} L\left(\mathcal{H}, \mathcal{K}_{i}\right) \cong L(\mathcal{H}, \mathcal{K})$. If we fix an injective $\mathcal{V}=\left\{V_{i}\right\}_{i \in \mathbb{I}_{m}} \in \mathcal{R} \mathcal{S}_{\mathcal{I}}(m, \mathbf{k}, d)$, then the map $\|\cdot\|_{\mathcal{V}}: L(m, \mathbf{k}, d) \rightarrow \mathbb{R}_{+}$given by

$$
\|\mathcal{W}\|_{\mathcal{V}} \stackrel{\text { def }}{=}\left\|E_{1}(\mathcal{V}, \mathcal{W})\right\|_{\infty}=\max _{i \in \mathbb{I}_{m}}\left\|W_{i}^{*} V_{i}\right\|_{2} \quad \text { for } \quad \mathcal{W}=\left\{W_{i}\right\}_{i \in \mathbb{I}_{m}} \in L(m, \mathbf{k}, d)
$$

is a norm in $L(m, \mathbf{k}, d)$. Indeed, the only non trivial condition is the faithfulness. But the fact that $\mathcal{V} \in \mathcal{R} \mathcal{S}_{\mathcal{I}}(m, \mathbf{k}, d)$ (i.e. $V_{i}$ is surjective for every $i \in \mathbb{I}_{m}$ ) assures that $\|\mathcal{W}\|_{\mathcal{V}}=0 \Longrightarrow$ $\left\|W_{i}^{*} V_{i}\right\|_{2}=0$ for every $i \in \mathbb{I}_{m} \Longrightarrow \mathcal{W}=0$.

Since $D(\mathcal{V})$ is closed in $L(m, \mathbf{k}, d)$ with the usual norm and all norms are equivalent on $L(m, \mathbf{k}, d)$, then $D(\mathcal{V})$ is $\|\cdot\|_{\mathcal{V}}$-closed in $L(m, \mathbf{k}, d)$. Therefore there exist elements $\mathcal{W} \in D(\mathcal{V})$ such that $\|\mathcal{W}\|_{\mathcal{V}}=\min _{\mathcal{N} \in D(\mathcal{V})}\|\mathcal{N}\|_{\mathcal{V}}=e_{1}(\mathcal{V})$. Indeed, the intersection of $D(\mathcal{V})$ with a fixed closed ball is a compact set. On the other hand, $D(\mathcal{V})$ is convex (actually it is an affine manifold). Since every norm is a convex map, we have proved the following result:

Proposition 12 Let $\mathcal{V} \in \mathcal{R S}_{\mathcal{I}}(m, \mathbf{k}, d)$ be an injective system. Then the set $D_{1}(\mathcal{V})$ of 1-loss optimal dual RS's for $\mathcal{V}$ is non-empty, compact and convex.

Theorem 13 Let $\mathcal{V}=\left\{V_{i}\right\}_{i \in \mathbb{I}_{m}} \in \mathcal{P}(m, \mathbf{k}, d)$ with weights $\mathbf{v}=\left(v_{i}\right)_{i \in \mathbb{I}_{m}}$. If

$$
\left\|S_{\mathcal{V}}^{-1} V_{i}^{*} V_{i}\right\|_{2}=v_{i}^{2}\left\|S_{\mathcal{V}}^{-1} P_{R\left(V_{i}^{*}\right)}\right\|_{2}=c \quad \text { for every } \quad i \in \mathbb{I}_{m}
$$

then $\mathcal{V}^{\#}$, the canonical dual $R S$ of $\mathcal{V}$, is the unique 1-loss optimal dual $R S$ for $\mathcal{V}$ (and hence the $r$-loss optimal dual $R S$ for every $r$ ). In other words, $D_{1}(\mathcal{V})=\left\{\mathcal{V}^{\#}\right\}$.

Proof. By Proposition 12 , there exists some $\mathcal{W}=\left\{W_{i}\right\}_{i \in \mathbb{I}_{m}} \in D_{1}(\mathcal{V})$. Then

$$
\|\mathcal{W}\|_{\mathcal{V}}=\max _{i \in \mathbb{I}_{m}}\left\|W_{i}^{*} V_{i}\right\|_{2} \leq \max _{i \in \mathbb{I}_{m}}\left\|S_{\mathcal{V}}^{-1} V_{i}^{*} V_{i}\right\|_{2}=\left\|\mathcal{V}^{\#}\right\|_{\mathcal{V}}=c
$$

If we denote each $V_{i} S_{\mathcal{V}}^{-1}=C_{i}$, then $\left\|W_{i}^{*} V_{i}\right\|_{2}^{2} \leq c=\left\|C_{i}^{*} V_{i}\right\|_{2}^{2}$ for every $i \in \mathbb{I}_{m}$. Recall that, by Eq. (3),$\left\|W_{i}^{*} V_{i}\right\|_{2}^{2}=v_{i}^{2}\left\|W_{i}^{*}\right\|_{2}^{2}$, since $V_{i} V_{i}^{*}=v_{i}^{2} I_{\mathcal{K}_{i}}$. Similarly, we get that each $\left\|C_{i}^{*} V_{i}\right\|_{2}^{2}=v_{i}^{2}\left\|C_{i}^{*}\right\|_{2}^{2}$. Therefore $\left\|W_{i}\right\|_{2}^{2} \leq\left\|C_{i}^{*}\right\|_{2}^{2}$ for every $i \in \mathbb{I}_{m}$. Note that

$$
\left\|W_{i}^{*}\right\|_{2}^{2}=\left\|C_{i}^{*}+\left(W_{i}^{*}-C_{i}^{*}\right)\right\|_{2}^{2}=\left\|C_{i}^{*}\right\|_{2}^{2}+\left\|W_{i}^{*}-C_{i}^{*}\right\|_{2}^{2}+2 \operatorname{Re}\left(\operatorname{tr}\left[\left(W_{i}^{*}-C_{i}^{*}\right) C_{i}\right]\right)
$$

and hence $\left\|W_{i}^{*}-C_{i}^{*}\right\|_{2}^{2}+2 \operatorname{Re}\left(\operatorname{tr}\left[\left(W_{i}^{*}-C_{i}^{*}\right) C_{i}\right]\right) \leq 0$, for every $i \in \mathbb{I}_{m}$. Finally,

$$
\sum_{i \in \mathbb{I}_{m}} \operatorname{tr}\left[\left(W_{i}^{*}-C_{i}^{*}\right) C_{i}\right]=\operatorname{tr}\left[\left(T_{\mathcal{W}}^{*}-T_{\mathcal{V} \#}^{*}\right) T_{\mathcal{V}} S_{\mathcal{V}}^{-1}\right]=0
$$


since both $\mathcal{W}$ and $\mathcal{V}^{\#}$ are dual RS's for $\mathcal{V}$. Then

$$
0 \leq \sum_{i \in \mathbb{I}_{m}}\left\|W_{i}^{*}-C_{i}^{*}\right\|_{2}^{2}=\sum_{i \in \mathbb{I}_{m}}\left\|W_{i}^{*}-C_{i}^{*}\right\|_{2}^{2}+\sum_{i \in \mathbb{I}_{m}} 2 \operatorname{Re}\left(\operatorname{tr}\left[\left(W_{i}^{*}-C_{i}^{*}\right) C_{i}\right]\right) \leq 0,
$$

which implies that $\mathcal{W}=\left\{W_{i}\right\}_{i \in \mathbb{I}_{m}}=\left\{C_{i}\right\}_{i \in \mathbb{I}_{m}}=\mathcal{V}^{\#}$.

A system $\mathcal{V} \in \mathcal{R S}(m, \mathbf{k}, d)$ is called a protocol for $\mathcal{H}$ if $S_{\mathcal{V}}=I_{\mathcal{H}}$. This notion appears in [4], 15] (see also [5], where protocols are related to $C^{*}$-encodings with noiseless subsystems).

Corollary 14 Let $\mathcal{V}=\left\{V_{i}\right\}_{i \in \mathbb{I}_{m}} \in \mathcal{P}(m, \mathbf{k}, d)$ be a projective protocol for $\mathcal{H}$ (i.e. $S_{\mathcal{V}}=I$ ) such that $\left\|V_{i}^{*} V_{i}\right\|_{2}=v_{i}^{2} k_{i}^{1 / 2}=c$ for every $i \in \mathbb{I}_{m}$. Then $D_{1}(\mathcal{V})=\left\{\mathcal{V}^{\#}\right\}=\{\mathcal{V}\}$.

Proof. By hypothesis $S_{\mathcal{V}}=I_{\mathcal{H}}$, and hence $\left\|S_{\mathcal{V}}^{-1} V_{i}^{*} V_{i}\right\|_{2}=\left\|V_{i}^{*} V_{i}\right\|_{2}=c$ for every $i \in \mathbb{I}_{m}$. Thus, the previous theorem can be applied in this case.

Remark 15 Examples of projective protocols as in Corollary 14 are the equi-dimensional uniform projective protocols i.e., $\left\{V_{i}\right\}_{i \in \mathbb{I}_{m}} \in \mathcal{P}(m, k \mathbb{1}, d)$ that are uniform. These are the analogues of the so-called uniform fusion frames.

The $(m, \mathbb{1}, d)$ case of Theorem 13 is a rephrasing of [14, Thm 2.6], since all vector frames are projective as RS's. Moreover, we can conclude from the examples [14, Section 3] that the optimal dual system $\mathcal{W} \in D_{1}(\mathcal{V})$ may not be the canonical dual RS and may be not unique for a general $\mathcal{V} \in \mathcal{P}(m, \mathbf{k}, d)$.

\section{Stability of RS's under erasures of coefficient packets}

In this section we consider a different approach to the erasures problem. Indeed given a fixed RS $\mathcal{V}$, assume that we can identify the set $J \subset \mathbb{I}_{m}$ such that $\left\{V_{i} x\right\}_{i \in J}$ are the missing or delayed packets of coefficients in the transmission of the signal $x \in \mathcal{H}$. In this case we shall state conditions which assure that the system $\mathcal{V}_{J}=\left\{V_{i}\right\}_{i \in \mathbb{I}_{m} \backslash J}$ corresponding to the remaining set of indexes is still a RS. Notice that this last fact allows to have perfect reconstruction with suitable dual RS's. Hence, an explicit computation of the canonical dual $\left(\mathcal{V}_{J}\right)^{\#}$ is given in this case.

The following statement is a generalization and a slight improvement of similar results of $\mathrm{P}$. Casazza and G. Kutyniok [7] and Asgari [1] for fusion frames.

Proposition 16 Let $\mathcal{V}=\left\{V_{i}\right\}_{i \in \mathbb{I}_{m}} \in \mathcal{R} \mathcal{S}(m, \mathbf{k}, d)$ with bounds $A_{\mathcal{V}}, B_{\mathcal{V}}$. Fix a subset $J \subset \mathbb{I}_{m}$ and consider the matrix $M_{J} \stackrel{\text { def }}{=} I_{d}-\sum_{i \in J} V_{i}^{*} V_{i} S_{\mathcal{V}}^{-1} \in \mathcal{M}_{n}(\mathbb{C})$. Then,

$$
\mathcal{V}_{J}=\left(V_{i}\right)_{i \in \mathbb{I}_{m} \backslash J} \quad \text { is a RS for } \quad \mathcal{H} \cong \mathbb{C}^{d} \Longleftrightarrow M_{J} \in \mathcal{G} l(d)
$$

In this case we can compute the following data for $\mathcal{V}_{J}$ :

1. The frame operator $S_{\mathcal{V}_{J}}=M_{J} S_{\mathcal{V}}$.

2. The bounds of $\mathcal{V}_{J}$ can be estimated by $\frac{A_{\mathcal{V}}}{\left\|M_{J}^{-1}\right\|} \leq A_{\mathcal{V}_{J}}$ and $B_{\mathcal{V}_{J}} \leq B_{\mathcal{V}}$.

3. The canonical dual can be characterized as

$$
\left(\mathcal{V}_{J}\right)^{\#}=\left\{V_{i} S_{\mathcal{V}_{J}}^{-1}\right\}_{i \notin J}=\left\{\mathcal{V}_{i}^{\#} M_{J}^{-1}\right\}_{i \notin J} \stackrel{\text { def }}{=}\left(\mathcal{V}^{\#}\right)_{J} \cdot M_{J}^{-1}
$$

That is, $\left(\mathcal{V}_{J}\right)^{\#}$ is the truncation of the canonical dual $\mathcal{V}^{\#}$ modified with $M_{J}^{-1}$. 
Proof. It is straightforward to check that $M_{J}=S_{\mathcal{V}_{J}} S_{\mathcal{V}}^{-1} \Longrightarrow S_{\mathcal{V}_{J}}=M_{J} S_{\mathcal{V}}$. This last fact implies the equivalence of Eq. (8). On the other hand,

$$
A_{\mathcal{V}}\left\|M_{J}^{-1}\right\|^{-1} \leq\left\|\left(M_{J} S_{\mathcal{V}}\right)^{-1}\right\|^{-1}=\left\|S_{\mathcal{V}_{J}}^{-1}\right\|^{-1}=A_{\mathcal{V}_{J}}
$$

The fact that $0<S_{\mathcal{V}_{J}} \leq S_{\mathcal{V}}$ assures that $B_{\mathcal{V}_{J}} \leq B_{\mathcal{V}}$. Let us denote $\mathcal{V}^{\#}=\left\{W_{i}\right\}_{i \in \mathbb{I}_{m}}$ and $\left(\mathcal{V}^{\#}\right)_{J}=\left\{W_{i}\right\}_{i \notin J}$. Then the formula $S_{\mathcal{V}_{J}}=M_{J} S_{\mathcal{V}}$ gives the equality

$$
\left(\mathcal{V}^{\#}\right)_{J} \cdot M_{J}^{-1} \stackrel{\text { def }}{=}\left\{W_{i} M_{J}^{-1}\right\}_{i \notin J}=\left\{V_{i} S_{\mathcal{V}}^{-1} M_{J}^{-1}\right\}_{i \notin J}=\left\{V_{i} S_{\mathcal{V}_{J}}^{-1}\right\}_{i \notin J}=\left(\mathcal{V}_{J}\right)^{\#}
$$

Remark 17 In [7] P. Casazza and G. Kutyniok stated the sufficient (and easily computable) condition $\sum_{i \in J}\left\|V_{i}\right\|_{s p}^{2}<A_{\mathcal{V}}$ for the invertibility of the matrix $M_{J}$ of Proposition 16. Indeed, if $\left\|\sum_{i \in J} V_{i}^{*} V_{i}\right\|_{s p}<A_{\mathcal{V}}$ (compare with the condition $\sum_{i \in J}\left\|V_{i}\right\|_{s p}^{2}<A_{\mathcal{V}}$ ), then $\left\|I_{d}-M_{J}\right\|_{s p}<1$ and $M_{J} \in \mathcal{G} l(d)$. They also give the estimation $A_{\mathcal{V}}-\sum_{i \in J}\left\|V_{i}\right\|_{s p}^{2} \leq A_{\mathcal{V}_{J}}$. This follows from

$$
A_{\mathcal{V}}-\sum_{i \in J}\left\|V_{i}\right\|_{s p}^{2} \leq A_{\mathcal{V}}-\left\|\sum_{i \in J} V_{i}^{*} V_{i}\right\|_{s p} \leq \frac{A_{\mathcal{V}}}{\left\|M_{J}^{-1}\right\|_{s p}} \stackrel{\text { Prop }}{\leq} A_{\mathcal{V}_{J}}
$$

The result of Asgari in [1] is similar to Eq. (8), but stated for fusion frames and assuming that $|J|=1$, with a different lower bound for $\mathcal{V}_{J}$.

\section{Approximation by projective RS's}

Given a fixed $\mathcal{V} \in \mathcal{P}(m, \mathbf{k}, d)$, notice that the optimal dual RS's obtained in Theorems 7, and 13 , are not projective RS's, in general. Although there could be some projective elements in $D(\mathcal{V})$ (we shall focus this problem in the following section), we are interested in those $(m, \mathbf{k}, d)$-projective RS's that are closest, with respect to some distance function, to a fixed $\mathcal{S}=\left\{S_{i}\right\}_{i \in \mathbb{I}_{m}} \in D(\mathcal{V})$ which has some desired properties. Given $\mathcal{W} \in \mathcal{R} \mathcal{S}(m, \mathbf{k}, d)$, we consider

$$
d(\mathcal{S}, \mathcal{W}) \stackrel{\text { def }}{=}\left\|T_{\mathcal{S}}-T_{\mathcal{W}}\right\|_{2}=\left\|T_{\mathcal{S}}^{*}-T_{\mathcal{W}}^{*}\right\|_{2}
$$

the distance between their synthesis (or analysis) operators. Hence, we seek for $\mathcal{W}_{0} \in$ $\mathcal{P}(m, \mathbf{k}, d)$ that minimize $d(\mathcal{S}, \mathcal{W})$ among the projective RS's. In what follows we will describe the structure of such (unique) minimizers in case $\mathcal{S}$ is an injective RS. As one would expect, its "directions" are the coisometries of the polar decompositions of the coordinate operators $S_{i}$ of $\mathcal{S}$, while its weights are the "averages" of their singular values. We need first some preliminary results:

Given $k, n \in \mathbb{N}$ such that $k \leq n$, we denote by

$$
\mathcal{I}(k, n)=\left\{U \in L\left(\mathbb{C}^{k}, \mathbb{C}^{n}\right): U^{*} U=I_{k}\right\}
$$

the set of isometries from $\mathbb{C}^{k}$ into $\mathbb{C}^{n}$. The following result can be found in [2]:

Lemma 18 Let $k, n \in \mathbb{N}$ such that $k \leq n$, and let $A \in \mathcal{M}_{n, k}(\mathbb{C})$ be a full rank matrix with polar decomposition $A=U|A|$ with $U \in \mathcal{I}(k, n)$. Then $\|A-U\|_{2}=\min _{V \in \mathcal{I}(k, n)}\|A-V\|_{2}$. 
Recall that for every $A \in \mathcal{M}_{n, k}(\mathbb{C})$ its polar decomposition satisfies

$$
A=U|A|=\left|A^{*}\right| U \Longrightarrow U^{*} A=|A| \quad \text { and } \quad A^{*}=U^{*}\left|A^{*}\right|,
$$

where $U \in \mathcal{M}_{n, k}(\mathbb{C})$ has $\operatorname{ker} U=\operatorname{ker} A$. As expected, the aproximation of a RS by a projective RS $\left\{S_{i}\right\}_{i \in \mathbb{I}_{m}}$ relies on the coisometries that best approximate each $S_{i}$, which are determined by the polar decomposition of $S_{i}$. Nevertheless, one has to determine the appropiate weights which combine those coisometries. That is done in the following Proposition.

Proposition 19 Let $\mathcal{S}=\left\{S_{i}\right\}_{i \in \mathbb{I}_{m}} \in \mathcal{R} \mathcal{S}_{\mathcal{I}}(m, \mathbf{k}, d)$. Then there exists a unique

$$
\mathcal{W}_{0} \in \mathcal{P}(m, \mathbf{k}, d) \quad \text { such that } \quad d\left(\mathcal{S}, \mathcal{W}_{0}\right)=\min _{\mathcal{W} \in \mathcal{P}(m, \mathbf{k}, d)} d(\mathcal{W}, \mathcal{S})
$$

and it is given by $\mathcal{W}_{0}=\left\{\alpha_{i} U_{i}\right\}_{i \in \mathbb{I}_{m}}$ where each $\alpha_{i}=\frac{\operatorname{tr}\left|S_{i}\right|}{k_{i}}$ and $S_{i}=U_{i}\left|S_{i}\right|$ is the polar decomposition of each $S_{i}$.

Proof. Let $\mathcal{W} \in \mathcal{P}(m, \mathbf{k}, d)$ be a system such that the minimum in (10) is attained at $\mathcal{W}$. Denote by $\mathbf{w}=\left(w_{i}\right)_{i \in \mathbb{I}_{m}} \in \mathbb{R}_{+}^{m}$ the weights of $\mathcal{W}$. Notice that

$$
\left\|T_{\mathcal{S}}^{*}-T_{\mathcal{W}}^{*}\right\|_{2}^{2}=\sum_{i=1}^{m}\left\|S_{i}^{*}-W_{i}^{*}\right\|_{2}^{2} \quad \text { and } \quad \text { each } \quad W_{i} W_{i}^{*}=w_{i}^{2} I_{\mathcal{K}_{i}},
$$

Thus, each isometry $w_{i}^{-1} W_{i}^{*} \in \mathcal{I}\left(k_{i}, d\right)$ attains the minimum in the optimization problem

$$
\left\|w_{i}^{-1} W_{i}^{*}-\frac{S_{i}^{*}}{w_{i}}\right\|_{2}=\min _{X \in \mathcal{I}\left(k_{i}, d\right)}\left\|X-\frac{S_{i}^{*}}{w_{i}}\right\|_{2},
$$

where, by hypothesis, each $w_{i}^{-1} S_{i}^{*}$ is a full rank linear transformation. By Lemma 18 we get that $w_{i}^{-1} W_{i}^{*}=U_{i}^{*}$, the isometry of the polar decomposition

$$
w_{i}^{-1} S_{i}^{*}=U_{i}^{*}\left|w_{i}^{-1} S_{i}^{*}\right|=w_{i}^{-1}\left(U_{i}^{*}\left|S_{i}^{*}\right|\right) \stackrel{(9)}{\Longrightarrow} S_{i}=U_{i}\left|S_{i}\right| \quad \text { and } \quad \operatorname{ker} U_{i}=\operatorname{ker} S_{i}
$$

and hence $W_{i}=w_{i} U_{i}$. Next we show that each $w_{i}=\frac{\operatorname{tr}\left|S_{i}\right|}{k_{i}}$. Fix $i \in \mathbb{I}_{m}$. Then

$$
\left\|S_{i}-w_{i} U_{i}\right\|_{2}=\min _{\alpha>0}\left\|S_{i}-\alpha U_{i}\right\|_{2}
$$

Therefore $w_{i} \cdot\left\|U_{i}\right\|_{2}$ is the norm of the orthogonal projection of $S_{i}$ to the line $\mathbb{R} U_{i}$, using the $\mathbb{R}$-inner product $\langle A, B\rangle=\operatorname{Re}\left[\operatorname{tr}\left(B^{*} A\right)\right]$. It can be computed explicitly:

$$
0 \leq \frac{\operatorname{tr}\left|S_{i}\right|}{\left\|U_{i}\right\|_{2}} \stackrel{\text { (9) }}{=} \frac{\operatorname{tr}\left(U_{i}^{*} S_{i}\right)}{\left\|U_{i}\right\|_{2}}=\left|\left\langle S_{i}, \frac{U_{i}}{\left\|U_{i}\right\|_{2}}\right\rangle\right|=\left\|P_{\mathbb{R} U_{i}}\left(S_{i}\right)\right\|_{2}=w_{i} \cdot\left\|U_{i}\right\|_{2},
$$

for every $i \in \mathbb{I}_{m}$. Then we obtain the equalities $w_{i}=\left\|U_{i}\right\|_{2}^{-2} \operatorname{tr}\left|S_{i}\right|=\frac{\operatorname{tr}\left|S_{i}\right|}{k_{i}}$. 


\section{Examples}

In this section we present a variety of examples related with the previous sections. First, we exhibit a family of (projective) RS's which satisfies the hypotheses of Corollary 9 and Theorem 13, the so-called group RS. In particular, the canonical dual of a group RS (which is also a group $\mathrm{RS}$ ) is the unique 1-loss optimal dual RS for the 2-error as well as for the WCRE. The remaining subsections are devoted to the study of particular cases of RS where $D(\mathcal{V}) \cap \mathcal{P}(m, \mathbf{k}, d) \neq \varnothing$. The first examples show that for certain projective systems we can explicitly construct projective duals, which in general will not coincide with the canonical duals. The last example describes a Riesz RS whose unique dual RS (i.e. the canonical dual) is not projective. This leads to a characterization for Riesz RS's with projective canonical dual.

\subsection{Group reconstruction systems}

We begin by rephrasing some basic notions and results from [13] in the RS's setting: Let $\mathcal{K}_{0} \cong \mathbb{C}^{k}$ and $V \in L\left(\mathcal{H}, \mathcal{K}_{0}\right)$. Given a unitary representation $G \ni g \mapsto U_{g} \in \mathcal{U}(\mathcal{H})$ of a finite group $G$ in $\mathcal{U}(\mathcal{H})$ we say that

$$
\mathcal{V}(G, V) \stackrel{\text { def }}{=}\left\{V U_{g}\right\}_{g \in G}
$$

is a $G$-reconstruction system (shortly, $G$-RS) if $\mathcal{V}(G, V) \in \mathcal{R} \mathcal{S}(m, k \mathbb{1}, d$ ), where $m=|G|$. In this case the space $\mathcal{K}=\mathcal{K}_{0}^{m}$. If $\mathcal{S}=V^{*}\left(\mathcal{K}_{0}\right)$, this is equivalent to the fact that

$$
\operatorname{span}\left\{\bigcup_{g \in G} U_{g}(\mathcal{S})\right\}=\mathcal{H}
$$

where $V \in L(\mathcal{H}, \mathcal{K})$ is the base operator for $\mathcal{V}(G, V)$. This definition of G-RS reduces to that of $G$-frame in the vector frames setting. Following [13] and [10] we state a series of properties of G-RS's whose proofs are similar to the frame case: Fix $V \in L\left(\mathcal{H}, \mathcal{K}_{0}\right)$.

1. Observe that the system $\mathcal{V}(G, V)$ is:

(a) Projective (and uniform) if in addition $V V^{*}=v^{2} I_{\mathcal{K}}$ for some $v>0$;

(b) Injective if in addition $V^{*}$ is injective, in which case also $R\left(V U_{g}\right)=\mathcal{K}$ for every $g \in G$.

2. Notice that the RS-operator of $\mathcal{V}(G, V)$ has the following structure:

$$
S_{G, V} \stackrel{\text { def }}{=} S_{\mathcal{V}(G, V)}=\sum_{g \in G} U_{g}^{*} V^{*} V U_{g}=\sum_{g \in G} U_{g^{-1}} V^{*} V U_{g} .
$$

3. The RS-operator $S_{G, V}$ (and therefore $S_{G, V}^{-1}$ ) commutes with the unitary representation of $G$ :

$$
U_{h} \cdot S_{G, V}=S_{G, V} \cdot U_{h} \quad \text { for every } \quad h \in G .
$$

4. In particular, the canonical dual of a $G$-RS is another $G$-RS:

$$
\mathcal{V}(G, V)^{\#}=\left\{V U_{g} S_{G, V}^{-1}\right\}_{g \in G}=\left\{V S_{G, V}^{-1} U_{g}\right\}_{g \in G}=\mathcal{V}\left(G, V S_{G, V}^{-1}\right)
$$


In order to apply our previous results, assume now that the base operator $V \in L(\mathcal{H}, \mathcal{K})$ satisfies $V V^{*}=v^{2} I_{\mathcal{K}}$. Then $\mathcal{V}(G, V)$ is an equi-dimensional uniform projective RS. Hence, Corollary 9 implies that the 1-loss optimal dual RS for $\mathcal{V}(G, V)$ for the 2-error is its canonical dual $\mathcal{V}(G, V)^{\#}$. In this case then $\mathcal{V}(G, V)$ also satisfies the hypothesis of Theorem 13: $\mathcal{V}(G, V)$ is a $G$-projective $\mathrm{RS}$, and

$$
\left\|\left(S_{G, V}^{-1} U_{g}^{*} V^{*}\right)\left(V U_{g}\right)\right\|_{2}=\left\|U_{g}^{*}\left(S_{G, V}^{-1} V^{*} V\right) U_{g}\right\|_{2}=\left\|S_{G, V}^{-1} V^{*} V\right\|_{2}=c .
$$

Thus, the canonical dual of such $G$-RS's is the unique 1-loss optimal dual for the worst-case error. If the base operator $V \in L(\mathcal{H}, \mathcal{K})$ is surjective, so that $\mathcal{V}(G, V)$ is an injective RS then, using Proposition 19, the projective RS nearest to $\mathcal{V}(G, V)^{\#}$ can be computed in the following way: For every $g \in G$, we have that

$$
\left|V S_{G, V}^{-1} U_{g}\right|^{2}=U_{g}^{*} S_{G, V}^{-1} V^{*} V S_{G, V}^{-1} U_{g}=U_{g}^{*}\left|V S_{G, V}^{-1}\right|^{2} U_{g}
$$

Taking square roots at both sides, we get that $\left|V U_{g} S_{G, V}^{-1}\right|=U_{g}^{*}\left|V S_{G, V}^{-1}\right| U_{g}$ for every $g \in G$. Therefore, if we consider the polar decomposition $V S_{G, V}^{-1}=W\left|V S_{G, V}^{-1}\right|$ of $V S_{G, V}^{-1}$, then also

$$
V S_{G, V}^{-1} U_{g}=\left(W U_{g}\right)\left(U_{g}^{*}\left|V S_{G, V}^{-1}\right| U_{g}\right)=\left(W U_{g}\right)\left|V U_{g} S_{G, V}^{-1}\right|
$$

is the polar decomposition of each entry $V S_{G, V}^{-1} U_{g}$ of $\mathcal{V}(G, V)^{\#}$. In conclusion, if we denote

$$
w=\frac{\operatorname{tr}\left|V S_{G, V}^{-1}\right|}{k}, \quad \text { then } \quad \mathcal{V}(G, w W)=\left\{w W U_{g}\right\}_{g \in G}
$$

is the best projective approximation of $\mathcal{V}(G, V)^{\#}$ provided by Proposition 19. It is clear from the previous computations that it is again a $G$-RS.

\subsection{Dual projective systems}

Next we consider an example of a system $\mathcal{V}=\left\{V_{i}\right\}_{i \in \mathbb{I}_{m}} \in \mathcal{P}(m, \mathbf{k}, d)$ with projective dual systems but such that $\mathcal{V}^{\#} \notin \mathcal{P}(m, \mathbf{k}, d)$ :

Example 20 Let $d=3, m=2$ and $\mathbf{k}=(2,2)$. Let $V_{1}$ and $V_{2} \in L\left(\mathbb{C}^{3}, \mathbb{C}^{2}\right)$ be given by

$$
V_{1}(x, y, z)=(y, z) \quad \text { and } \quad V_{2}(x, y, z)=(x, z) \quad \text { for every } \quad(x, y, z) \in \mathbb{C}^{3} .
$$

Then $\mathcal{V}=\left(V_{1}, V_{2}\right) \in \mathcal{P}(m, \mathbf{k}, d)$ with weights $\mathbb{1}_{2}$. If $\mathcal{S}_{1}=\left\{e_{1}\right\}^{\perp}$ and $\mathcal{S}_{2}=\left\{e_{2}\right\}^{\perp}$, then

$$
S_{\mathcal{V}}=V_{1}^{*} V_{1}+V_{2}^{*} V_{2}=P_{\mathcal{S}_{1}}+P_{\mathcal{S}_{2}}=\left(\begin{array}{ccc}
1 & 0 & 0 \\
0 & 1 & 0 \\
0 & 0 & 2
\end{array}\right) \text {. }
$$

Therefore $\mathcal{V}^{\#} \notin \mathcal{P}(m, \mathbf{k}, d)$ since $S_{\mathcal{V}}^{-1} V_{1}^{*}(u, v)=\left(0, u, \frac{v}{2}\right)$ for $(u, v) \in \mathbb{C}^{2}$, so that the entry $V_{1} S_{\mathcal{V}}^{-1}$ of $\mathcal{V}^{\#}$ is not a multiple of a coisometry.

Let $\mathcal{W}=\left(W_{1}, W_{2}\right) \in \mathcal{P}(m, \mathbf{k}, d)$ and assume that $T_{\mathcal{W}}^{*} T_{\mathcal{V}}=W_{1}^{*} V_{1}+W_{2}^{*} V_{2}=I_{3}$. Denote by $\left\{v_{1}, v_{2}\right\}$ the canonical basis of $\mathbb{C}^{2}$ and by $\left\{e_{1}, e_{2}, e_{3}\right\}$ that of $\mathbb{C}^{3}$. Then, easy computations using the definition of $\mathcal{V}$ show that

$$
e_{3}=W_{1}^{*} v_{2}+W_{2}^{*} v_{2} \quad, \quad e_{2}=W_{1}^{*} V_{1} e_{2}=W_{1}^{*} v_{1} \quad \text { and } \quad e_{1}=W_{1}^{*} V_{2} e_{1}=W_{2}^{*} v_{1} .
$$


The last two equalities show that both $W_{1}^{*}$ and $W_{2}^{*}$ should be isometries with weight 1 . But in this case $\left\|W_{1}^{*} v_{2}\right\|=\left\|W_{2}^{*} v_{2}\right\|=1$ and their sum also has norm one. Let $\omega=\frac{1}{2}+i \frac{\sqrt{3}}{2}$. Then $|\omega|=1$ but $\omega+\bar{\omega}=1$. Then we can define $W_{1}^{*}, W_{2}^{*} \in L\left(\mathbb{C}^{2}, \mathbb{C}^{3}\right)$ by

$$
W_{1}^{*}(x, y)=(0, x, \omega y) \quad \text { and } \quad W_{2}^{*}(x, y)=(x, 0, \bar{\omega} y) \quad \text { for every } \quad(x, y) \in \mathbb{C}^{2} .
$$

The $W_{i}^{*}$ are isometries and satisfy the three conditions of (12). Therefore, the system $\mathcal{W}=\left(W_{1}, W_{2}\right)$ lies in $\mathcal{P}(m, \mathbf{k}, d)$ and it is a dual-RS for $\mathcal{V}$.

Nevertheless, if we consider $V_{1}$ and $V_{2}$ as operators in $L\left(\mathbb{R}^{3}, \mathbb{R}^{2}\right)$, then such a $\mathcal{W}$ can not exists in the real case. Indeed, looking at Eq. (12), we can deduce that $W_{1}^{*} v_{2} \in\left\{e_{2}\right\}^{\perp}$ and $W_{2}^{*} v_{2} \in\left\{e_{1}\right\}^{\perp}$. These facts, together with the equality $e_{3}=W_{1}^{*} v_{2}+W_{2}^{*} v_{2}$ imply that both $W_{1}^{*} v_{2}$ and $W_{2}^{*} v_{2} \in \operatorname{span}\left\{e_{3}\right\}$, which is impossible in the real case.

Example 21 We can generalize Example 20 in the following way: Assume that the projective system $\mathcal{V}=\left\{V_{i}\right\}_{i \in \mathbb{I}_{m}} \in \mathcal{P}(m, \mathbf{k}, d)$ has the property that all the projections $P_{i}=P_{R\left(V_{i}^{*}\right)}$ are pairwise commuting. In this case $D(\mathcal{V}) \cap \mathcal{P}(m, \mathbf{k}, d) \neq \varnothing$.

Indeed, suppose first that all the weights of $\mathcal{V}$ are 1 . Then $S_{\mathcal{V}}=\sum_{i \in \mathbb{I}_{m}} P_{i}$. The commutation hypothesis assures that, by taking all the possible intersections among the ranges of the projections $P_{i}$, we get a family of projections $\left(Q_{j}\right)_{j \in \mathbb{I}_{n}}$ such that

1. $Q_{i} Q_{j}=0$ if $i \neq j$ and $\sum_{j \in \mathbb{I}_{n}} Q_{j}=I_{\mathcal{H}}$.

2. $S_{\mathcal{V}}=\sum_{j \in \mathbb{I}_{n}} r_{j} Q_{j}$ with $r_{j} \in \mathbb{I}_{m}$ for every $j \in \mathbb{I}_{n}$.

3. For every $i \in \mathbb{I}_{m}$ there exists $\mathbb{J}_{i} \subseteq \mathbb{I}_{n}$ such that $P_{i}=\sum_{j \in \mathbb{J}_{i}} Q_{j}$.

We construct the system $\mathcal{W}=\left\{W_{i}\right\}_{i \in \mathbb{I}_{m}} \in D(\mathcal{V}) \cap \mathcal{P}(m, \mathbf{k}, d)$ as follows: let $W_{i}=V_{i} U_{i}$, where

$$
U_{i}=\sum_{j \in \mathbb{J}_{i}} \varepsilon_{i j} Q_{j} \quad \text { for some } \quad \varepsilon_{i j} \in\{1, \quad-1, \omega, \bar{\omega}\} \quad, \quad \text { where } \quad \omega=\frac{1}{2}+i \frac{\sqrt{3}}{2} .
$$

Note that since all $\left|\varepsilon_{i j}\right|=1$, then $\mathcal{W} \in \mathcal{P}(m, \mathbf{k}, d)$. A careful selection of these coefficients, taking account the parity of the numbers $r_{j}=\left|\mathbb{S}_{j}\right|$, where $\mathbb{S}_{j}=\left\{i \in \mathbb{I}_{m}: j \in \mathbb{I}_{i}\right\}$ for $j \in \mathbb{I}_{n}$, allows to find such a $\mathcal{W}$ such that $\mathcal{W} \in D(\mathcal{V})$. The general case follows from the previous case. Indeed, if $\mathcal{V}$ has weights $\mathbf{v}=\left(v_{i}\right)_{i \in \mathbb{I}_{m}}$, we replace the previous $\mathcal{W}$ by $\mathcal{W}_{\mathbf{v}}=\left\{v_{i}^{-2} W_{i}\right\}_{i \in \mathbb{I}_{m}}$.

Remark 22 (Projective dual pairs) The previous example gives a method to construct pairs of projective RS's $(\mathcal{V}, \mathcal{W})$ such that $\mathcal{W} \in D(\mathcal{V})$. Moreover, this method shows that for every choice of parameters $(m, \mathbf{k}, d)$ such that $\sum_{i \in \mathbb{I}_{m}} k_{i} \geq d$ there exist $\mathcal{V}, \mathcal{W} \in \mathcal{P}(m, \mathbf{k}, d)$ such that $\mathcal{W} \in D(\mathcal{V})$. Indeed, to find such a projective dual pair $(\mathcal{V}, \mathcal{W})$ we construct $\mathcal{V} \in \mathcal{P}(m, \mathbf{k}, d)$ in such a way that the projections $P_{i}=V_{i}^{*} V_{i}$ for $i \in \mathbb{I}_{m}$ are pairwise commuting (i.e. that are simultaneously diagonalizable by an orthonormal basis of $\mathcal{H}$ ). Then, we can apply the construction in the example above to obtain explicitly the projective dual $\mathcal{W}$.

These facts show that projective dual pairs are indeed more frequent than projective protocols i.e. RS's $\mathcal{V}$ such that $(\mathcal{V}, \mathcal{V})$ is a projective dual pair, since it is known that there are choices of parameters $(m, \mathbf{k}, d)$ for which no projective $(m, \mathbf{k}, d)$ - protocols exist (see [17, Example 3.1.2.]). 


\subsection{Riesz reconstruction systems}

The elements of $\mathcal{R} \mathcal{S}(m, \mathbf{k}, d)$ are called Riesz RS's if $\operatorname{dim} \mathcal{K}=\operatorname{tr} \mathbf{k}=d=\operatorname{dim} \mathcal{H}$. In this case, every $\mathcal{V}=\left\{V_{i}\right\}_{i \in \mathbb{I}_{m}} \in \mathcal{R} \mathcal{S}(m, \mathbf{k}, d)$ has the property that both the synthesis operator $T_{\mathcal{V}}$ and the analysis operator $T_{\mathcal{V}}^{*}$ are invertible. Also $T_{\mathcal{V} \#}^{*}=S_{\mathcal{V}}^{-1} T_{\mathcal{V}}^{*}=T_{\mathcal{V}}^{-1}$, and

$$
\mathcal{H}=R\left(V_{1}^{*}\right) \oplus \cdots \oplus R\left(V_{m}^{*}\right) \quad \text { (direct sum, but not necessarily orthogonal), }
$$

because the sum gives always $\mathcal{H}$ but in this case the sum must be direct by dimensional reasons. On the other hand, if $\mathcal{V}=\left\{V_{i}\right\}_{i \in \mathbb{I}_{m}} \in \mathcal{R} \mathcal{S}(m, \mathbf{k}, d)$ is a Riesz $\operatorname{RS}$ for $\mathcal{H}$ then

$$
D(\mathcal{V})=\left\{\mathcal{V}^{\#}\right\}
$$

since the only left inverse of $T_{\mathcal{V}}$ is $T_{\mathcal{V}}^{-1}=T_{\mathcal{V} \#}^{*}$. Recall that $T_{\mathcal{W}}^{*} T_{\mathcal{V}}=I_{\mathcal{H}}$ for every $\mathcal{W} \in D(\mathcal{V})$, and that the map $\mathcal{R} \mathcal{S}(m, \mathbf{k}, d) \ni \mathcal{W} \mapsto T_{\mathcal{W}}^{*}$ is one to one.

Example 23 Let $d=4, m=2$ and $\mathbf{k}=(2,2)$. We now construct a (necessarily) Riesz $\mathcal{V} \in \mathcal{P}(m, \mathbf{k}, d)$ such that $D(\mathcal{V}) \cap \mathcal{P}(m, \mathbf{k}, d)=\varnothing$. Let $V_{1}, V_{2} \in L\left(\mathbb{C}^{4}, \mathbb{C}^{2}\right)$ be given by

$$
V_{1}\left(x_{1}, x_{2}, x_{3}, x_{4}\right)=\left(x_{1}, x_{2}\right) \quad \text { and } \quad V_{2}\left(x_{1}, x_{2}, x_{3}, x_{4}\right)=\left(x_{3}, \frac{x_{2}-x_{4}}{\sqrt{2}}\right),
$$

for $\left(x_{1}, x_{2}, x_{3}, x_{4}\right) \in \mathbb{C}^{4}$. It is easy to see that $\mathcal{V}=\left(V_{1}, V_{2}\right) \in \mathcal{P}(m, \mathbf{k}, d)$ with weights $(1,1)$. Let us denote by $S=\operatorname{ker} V_{2}=\operatorname{span}\left\{e_{1}, e_{2}+e_{4}\right\} \subseteq \mathbb{C}^{4}$. Given $\mathcal{W} \in D(\mathcal{V})$, the equality

$$
W_{1}^{*} V_{1}+W_{2}^{*} V_{2}=I_{\mathcal{H}}
$$

implies that $W_{1}^{*} V_{1} x=x$ for every $x \in S$. Then $W_{1}^{*} \in L\left(\mathbb{C}^{2}, \mathbb{C}^{4}\right)$ is completely determined as the inverse of $\left.V_{1}\right|_{S}: S \rightarrow \mathbb{C}^{2}$. But we have that

$$
\left\|V_{1} e_{1}\right\|=\left\|e_{1}\right\|=1 \quad \text { while } \quad\left\|V_{1}\left(e_{2}+e_{4}\right)\right\|=\left\|e_{2}\right\|=\frac{\left\|e_{2}+e_{4}\right\|}{\sqrt{2}} .
$$

Then $\left.V_{1}\right|_{S}$ is not a multiple of an isometry and neither is $W_{1}^{*}$.

We can enlarge the previous example in order to get a RS with redundancy and without projective duals. Indeed, consider $\mathcal{V}_{0}=\left(V_{1}, V_{2}, V_{3}\right) \in \mathcal{P}(3,(2,2,2), 4)$ obtained from $\mathcal{V}$ by adding any coisometry $V_{3} \in L\left(\mathbb{C}^{4}, \mathbb{C}^{2}\right)$ such that also ker $V_{3}=\mathcal{S}$. Then, arguing as before, we conclude that there is no $\mathcal{W}=\left(W_{1}, W_{2}, W_{3}\right) \in D\left(\mathcal{V}_{0}\right)$ such that $W_{1}$ is a multiple of a coisometry.

Remark 24 Assume that $\mathcal{V}=\left\{V_{i}\right\}_{i \in \mathbb{I}_{m}} \in \mathcal{R S}(m, \mathbf{k}, d)$ is a Riesz RS. Then, arguing as in the previous example, it is easy to see that the following conditions are equivalent:

1. $D(\mathcal{V}) \cap \mathcal{P}(m, \mathbf{k}, d) \neq \varnothing$.

2. $\mathcal{V}^{\#} \in \mathcal{P}(m, \mathbf{k}, d)$.

3. If we denote by $S_{i}=\bigcap_{j \neq i} \operatorname{ker} V_{i}=\left(\bigoplus_{j \neq i} R\left(V^{*}\right)\right)^{\perp}$, then $\left.V_{i}\right|_{S_{i}} \in L\left(S_{i}, \mathcal{K}_{i}\right)$ is a multiple of an isometry, for every $i \in \mathbb{I}_{m}$.

These conditions are fulfilled if the sum of Eq. (13) is orthogonal. Also if every $k_{i}=1$. But there exist other cases in which $D(\mathcal{V}) \cap \mathcal{P}(m, \mathbf{k}, d) \neq \varnothing$. For example, if we take the operator $V_{1}$ of Eq. (15), and consider

$$
V_{3}\left(x_{1}, x_{2}, x_{3}, x_{4}\right)=\left(x_{1}-x_{3}, x_{2}-x_{4}\right) \quad \text { for } \quad\left(x_{1}, x_{2}, x_{3}, x_{4}\right) \in \mathbb{C}^{4},
$$

then the condition 3 is satisfied by $\mathcal{V}^{\prime}=\left(V_{1}, V_{3}\right) \in \mathcal{P}(2,(2,2), 4)$. 


\section{References}

[1] Asgari, Mohammad Sadegh, New characterizations of fusion frames (frames of subspaces). Proc. Indian Acad. Sci. Math. Sci. 119 (2009), no. 3, 369-382

[2] Bhatia, Rajendra, Matrix analysis, xii+347 pp. Graduate Texts in Mathematics, 169, Springer-Verlag, New York, (1997).

[3] J.J. Benedetto, M. Fickus, Finite normalized tight frames. Adv. Comput. Math. 18, No. 2-4, $357-385$ (2003).

[4] B.G. Bodmann, Optimal linear transmission by loss-insensitive packet encoding Appl. Comput. Harmon. Anal. 22 , no. 3, 274-285, (2007).

[5] B.G. Bodmann, D.W. Kribs, V.I. Paulsen Decoherence-Insensitive Quantum Communication by Optimal C*Encoding, IEEE Transactions on Information Theory 53, 4738-4749, (2007)

[6] B.G. Bodmann, V.I. Paulsen, Frames, graphs and erasures, Linear Algebra Appl. 404, 118-146, (2005).

[7] P.G. Casazza, G. Kutyniok, Robustness of Fusion Frames under Erasures of Subspaces and of Local Frame Vectors. Contemp. Math. 464, 149-160, (2008).

[8] P.G. Casazza, G. Kutyniok, S. Li, Fusion Frames and Distributed Processing. Appl. Comput. Harmon. Anal. 25, No. 1, 114-132, (2008).

[9] P.G. Casazza, G. Kutyniok, S. Li, C.J. Rozell, Modeling Sensor Networks with Fusion Frames. Proc. SPIE 6701, 67011M-1-67011M-11, (2007)

[10] D. Han, Classification of finite group-frames and super-frames, Canad. Math. Bull. 50, 85 -96,(2007).

[11] R.B. Holmes, V.I. Paulsen, Optimal frames for erasures, Linear Algebra Appl. 377, 31-51, (2004).

[12] G. Kutyniok, A. Pezeshki, R. Calderbank, T. Liu, Robust Dimension Reduction, Fusion Frames, and Grassmannian Packings. Appl. Comput. Harmon. Anal. 26, No. 1, 64-76, (2009).

[13] D. Han, D.R. Larson, Frames, bases and group representations. Mem. Amer. Math. Soc. 147, no. 697 (2000).

[14] J. Lopez, D. Han, Optimal dual frames for erasures, Linear Algebra Appl. 432, no. 1, 471-482, (2010).

[15] P. Massey, Optimal reconstruction systems for erasures and for the q-potential. Linear Algebra Appl. 431, no. 8, 1302-1316, (2009).

[16] P. Massey and M. Ruiz, Minimization of convex functionals over frame operators, Adv Comput Math 32, no. 2, 131-153, (2010).

[17] P. Massey, M. Ruiz and D. Stojanoff, The structure of minimizers of the frame potential on fusion frames, J. Fourier Analysis and Applications, 16 , no. 4, 514-543, (2010).

[18] P. Massey, M. Ruiz and D. Stojanoff, Duality in reconstruction systems, Linear Algebra Appl., in press.

[19] T. Strohmer and R.W Heath Jr., Grassmannian frames with applications to coding and communication, Appl. Comput. Harmon. Anal. 14, 257-275, (2003).

[20] W. Sun, G-frames and g-Riesz bases, J. Math. Anal. Appl. 322, No. 1, 437-452, (2006). 